\title{
Planar Construction
}

\section{of Extraordinary Magnetoresistance Sensor}

\author{
S. El-Ahmar ${ }^{a *}$, A.A. Pozniak ${ }^{a}$, J. JAnkowski $^{a}$, M. OszWAldowski ${ }^{b}$ \\ ${ }^{a}$ Poznan University of Technology, Nieszawska 13a, 60-965 Poznan, Poland \\ ${ }^{b}$ Industrial Research Institute for Automation and Measurements, Al. Jerozolimskie 202, 02-486 Warsaw, Poland
}

\begin{abstract}
A new version of the construction of the extraordinary magnetoresistance effect (EMR) based magnetic sensor has been proposed [2]. The differences between the original three dimensional (3D) construction and proposed 2D (planar) construction are presented. In proposed construction the metallic thin film (shunt) is coplanar with the semiconductor sensitive element. There are advantages of that planar construction like easier way of technological obtaining of the device. Another advantage is its application for EMR sensors based on new electronic materials like graphene and topological insulator thin films. The validity of the planar construction has been experimentally confirmed for model EMR sensors based on InSb/Ag structures. Comparison of the obtained experimental data with computational simulations of the EMR effect on planar model EMR sensors is performed Finite element method (FEM) is used as a tool for obtaining EMR effect simulations.
\end{abstract}

PACS: 85.80.Jm, 85.70.Kh, 73.43.Qt, 72.15. Gd

\section{Introduction}

The effect of extraordinary magnetoresistance (EMR) as well as potential practical application of EMR based sensors has been given in a review article by S. Solin [1]. The article just as many others suggested that EMR sensors were a great hope for very important applications in the magnetic read heads [1]. These sensors were expected to substitute GMR (giant magnetoresistance) based read heads which are in common use. That hope has not yet been fulfilled because of technological problems. The standard (3D) construction of EMR device proposed by Solin [1] demands advanced technology of preparation. We propose new planar (2D) construction which is not subject to such demand. These two possible constructions are shown on Fig. 1.
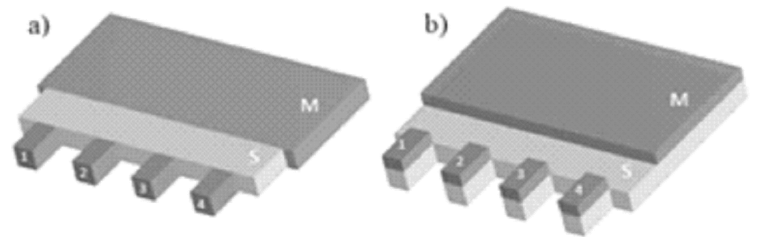

Fig. 1. Two configurations of EMR sensor, a) standard (3D) construction. b) proposed planar (2D) construction, $\mathrm{M}$ - highly conductive metal, $\mathrm{S}$ - high electron mobility semiconductor, $1-4$ are electric terminals.

There is a very simple principle of operation of the device shown in Fig. 1a. The current $J_{1,4}$ is injected

\footnotetext{
* corresponding author; e-mail: semir.el-ahmar@put.poznan.pl
}

through terminals 1 and 4 , and the voltage, $U_{2,3}$ is measured at terminals 2 and 3 . The sensor resistance is defined as $R=\left(U_{2,3}\right) / J_{1,4}$. In the absence of magnetic field measured voltage $U_{2,3}$ is very low because the current flows mainly through the metal which shunts the semiconductor. Consequently device resistance, $R(0)$ is very low. In the presence of magnetic field the current is partly ejected from the metal it starts to flow in semiconductor. In a result the voltage, $U_{2,3}$ and consequently $R(B)$ increases. The magnetoresistance signal is defined as follow: $M(B)=[R(B)-R(0)] / R(0)$. In that case the signal is biggest for high $R(B)$ and low $R(0)$. The construction of the device which operates in this way (shown in Fig. 1a) is based on specific semiconductor - metal (S-M) hybrid structure, composed of high electron mobility semiconductor and highly conductive metal. The most characteristic areas of that construction are sidewall connections between semiconductor and metal shunt as well as between semiconductor and metal terminals. Because of need of realization such connections the whole construction process is technologically demanding, difficult and liable to become automated. Therefore, a question may arise if it is possible to change the construction of the EMR sensor to make it technologically more affordable. Proposed sensor construction shown in Fig. 1b is the solution of the problem. The electrical equivalence of structures shown in Fig. 1a and b cannot be, from the point of view of the EMR effect, easily proved on a purely physical ground but it can be understood intuitively. In the proposed project we will prove that equivalence on experimental ground as well as by computational simulations. To intuitively comprehend the equivalence of both structures, it is necessary to recognize that in both case the metallic shunt plays effectively the same role. In the 
case of the structure shown in Fig. 1b, the shunt is placed on the top of the semiconductor layer. In the planar configuration of the EMR sensor, shown in Fig. 1b, the voltage and the current electrodes are placed on the top of lateral appendixes of the semiconductor layer. With such a solution, the shunt and the electrodes can be deposited at the same metal deposition process. Thereby, the planar construction of the sensor can entirely be realized in the standard thin film technology. Proposed new construction of EMR sensor greatly simplifies its manufacturing. The preliminary FEM simulations of EMR effect on model device in planar construction opens new possibilities of sensor parameters optimization.

\section{Sample preparation}

The process of easily obtaining EMR devices in planar configuration from standard Hall effect measurements samples will be presented. The semiconductor part of the investigated EMR structures is InSb layer epitaxially grown on semi-insulating GaAs substrate. The layer thickness is about $1 \mu \mathrm{m}$, and the substrate plate thickness is about $300 \mu \mathrm{m}$. Details of the preparation method of the epitaxial InSb layers and their basic electrical properties are given in Ref. [5]. The InSb film is shaped photolithographically to a form suitable for precise determination of the film electrical parameters as shown in Fig. 2a. The InSb film rests on GaAs substrate having the dimensions of $12 \times 8 \mathrm{~mm}^{2}$.

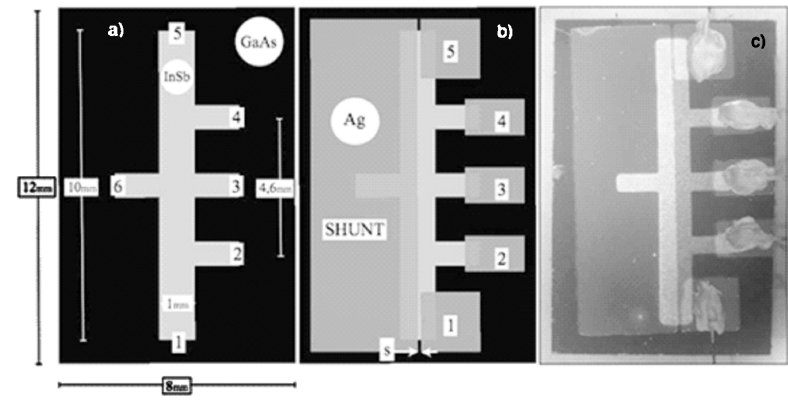

Fig. 2. Measurement structures for determination of electrical parameters. 1-6 are electric terminals, a) shows shape and dimensions of structure for determining electron concentration and mobility in InSb layers from Hall effect measurements, b) shows schematically planar structure for determining EMR effect, c) shows practical implementation of $b$ ) applied in present paper (optical microscope).

The InSb thin films designed for the Hall effect measurements have four lateral appendixes that form the terminals 2, 3, 4, and 6 in Fig. 2a. The terminals 1 and 5 are used for the driving current, $I$ injection. The terminals 2 and 4 are used for the determination of the voltage $U R$, from which the sample resistance $R(=U R / I)$ is determined. The Hall voltage $U H$ is measured on the terminals 3 and 6 . The measurements of $U R$ and $U H$ are performed by the application of spring electric contacts to the ends of the terminals $1-6$. From these measurements the electron concentration $n$ and the electron mobility $\mu$ of InSb films are determined using the following formula:

$$
\begin{aligned}
n & =\frac{I B}{e d U H} \\
\mu & =\frac{l}{w} \frac{U H}{I B R}
\end{aligned}
$$

where $B$ is the value of the applied magnetic induction, $e$ is the electron charge, $d$ is the film thickness and $l / w$ is the InSb film length-to-width ratio. In the present case $l / w=4$ (Fig. 2a). Equations (1) and (2) hold for $n$-type semiconductors. The electric parameters of the $n$-type thin film discussed in detail in this paper (sample no. 511) are as follows: specific resistance $\rho=3.8 \times 10^{-3} \Omega \mathrm{cm}$, electron concentration $n=0.9 \times 10^{17} \mathrm{~cm}^{-3}$, electron mobility $\mu=18000 \mathrm{~cm}^{2} / \mathrm{Vs}$, resistance $R=158 \Omega$, EMR sensor resistances at $B=0$ for $(15,24)-R_{15,24}(0)=$ $0.25 \Omega$ and for $(14,35)-R_{14,35}(0)=0.03 \Omega$. The ratio $\beta=R_{15,24}(0) / R=0.0016$.

After the determination of its electric parameters, the InSb thin film was equipped with metallic electrodes at the ends of the terminals and also with the shunt, as shown in Fig. 2b. This is done by the vacuum evaporation of about $1 \mu \mathrm{m}$ silver layer on the top of $\mathrm{InSb}$ layer. Measurements of the EMR effect are carried out on such prepared InSb-Ag hybrid structures equipped with copper leads attached to the electrodes with silver paste, as shown in Fig. 2c. Taking into account that a large EMR effect occurs only in the hybrid heterostructures containing high electron mobility materials, it is concluded that the film of Table is not an optimum InSb material for obtaining large EMR effect.

\section{Investigation of EMR effect}

The investigation results for sample $51-1$ are presented. The same sample is measured in two different terminal configurations named: symmetric and asymetric. These configurations differ with current injection and voltage measurement points. Such a terminal configuration where the current flows through terminals $i$ and $j$, and the voltage is measured at terminals $k$ and $l$ will be denoted as $(i j, k l)$. Therefore, in reference to Fig. 1a the symmetric configuration is $(14,23)$ and in reference to Fig. $2 \mathrm{~b}$ it is $(15,24)$. In the case when the terminals $k$ and $l$ are not symmetrically aligned in reference to terminals $i$ and $j$, the configuration is called asymmetric configuration. Examples of such asymmetric configurations (in reference to Fig. $2 \mathrm{~b})$ are $(15,23)$ and $(15,34)$. Recently, configurations described as IUIU or UIUI have been investigated [5]. In these configurations one voltage terminal $(U)$ lies between the current terminals $(J)$ or vice versa. Examples of such configurations are $(14,25)$ and $(14,35)$. The field dependence of the EMR for sample 51-1 measured in symmetric and asymmetric configuration is shown in Fig. 3. This experimental dependence was compared with the results of the numerical experiment conducted by means finite element simulations. 
The material parameters were taken as these of sample 51 - 1. The idea was to model the EMR phenomenon using two-dimensional (plane) geometry. This approach requires less computational power and is not as timeconsuming as the three-dimensional modeling. Once the geometry of the plane model was prepared, the physical discretization of the domain (meshing) was done (see Fig. 4). The mesh consisted of triangle Lagrange elements of second order which means that second order polynomials were used as the test functions. The density of the mesh was non-uniform due to rapid change of material parameters. Near the interface between two different materials which electric constants varied significantly, the mesh has to be finer (densificated) to prevent from potentially possible numerical errors. To enforce the current flow through the model, boundary conditions of the special type, namely terminal type, were applied. In a certain single point in the model the ground point condition was applied as well to provide the reference potential to the model. The influence of the external magnetic field was introduced by parameter modifying the conductivity tensor as described by Holz [3].

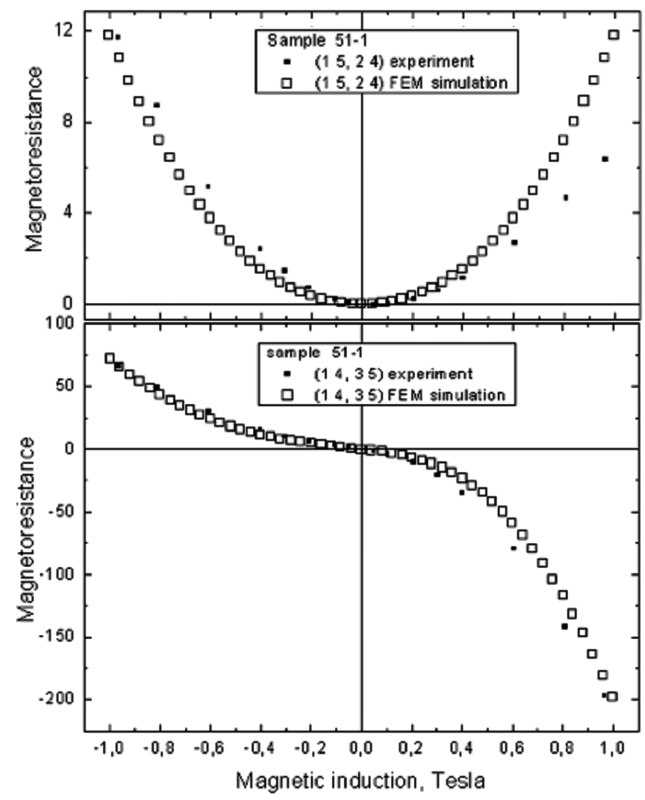

Fig. 3. Experimental magnetoresistance of sample $51-1$ in $(15,24)$ - top, and $(14,35)$ - bottom, terminal configurations at room temperature compared with FEM simulations.

The MR in the symmetric, $(15,24)$ configuration shows asymmetry associated with the magnetic field polarization. The asymmetry is of such a form that the MR is independent on the magnetic field polarization but the magnitude of MR depends on the field polarization. The FEM calculations (Fig. 3 - top) do not revealed such asymmetry which is also confirmed in literature $[3,7,8,9]$, however some experimental data [4] did reveal the existence of such asymmetry. However, the experimentally observed asymmetry was very small. We think

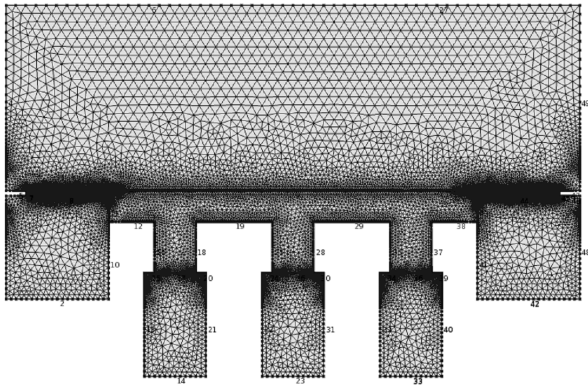

Fig. 4. Model of the sample 51-1 used for FEM simulations.

that the existence of the small asymmetry in our samples can tentatively ascribed to occurring electrical inhomogeneities that are expected to exist in them.

The MR can be positive for one magnetic field polarization, and negative for the opposite polarization. Such type of asymmetry associated with magnetic field polarization occurs, as a rule, in the case of the IUIU type configurations. For those configurations, the MR is very large, at least for one magnetic field polarization, and is larger than that observed for the other configurations. This is in a qualitative agreement with the MR effect magnitude in the IUIU FEM calculations (Fig. 3) as well as with earlier conclusions $[6,8,9]$. It results from the FEM analysis that the large magnitude of the EMR effect and the change of its sign with the magnetic field polarization for the IUIU configuration is the effect of interference of the MR and Hall effects in the S-M heterostructure. It may be observed in the Fig. 3 that in the $(14,35)$ configuration the absolute values of the MR may really be very high, reaching the value of 200 or more at $B=1 T$. Those values may be considered as very high taking into account that the electrical parameters of the InSb films are rather poor for obtaining a high MR, the measurements are performed at high (room) temperature, and sample construction is probably far from the optimum.

\section{Summary and conclusions}

In this work we present a new version of the construction of the EMR effect based magnetic field sensor. The experimental results are compared with FEM simulations. The difference between the standard 3D construction and the proposed new 2D planar construction of the sensor is explained in Fig. 1, and the actual planar construction of the sensor investigated here is shown in Fig. 2. An important advantage of the construction of the EMR sensor in the planar form is that it circumvents the technological problems of production that are characteristic for the standard 3D form. Therefore, the planar form solves considerably the problem of effective production of the magnetic sensors as well as the problem of an easy access to the material samples for the experimental investigation of the EMR effect. At present, because of 
the technological difficulties in the sample preparation, most of the literature information on the behaviour of EMR heterostructures is gained from the computer simulations, e.g. $[3,6,7,8-10]$. The planar configuration of the EMR heterostructure also solves the problem of the investigation and practical application of the EMR effect in the case of the new thin film materials like graphene and the topological insulators. Those materials having very high charge carrier mobility are very perspective for the construction of the galvanomagnetic devices including the magnetoresistors. When we have to deal with single or several monolayers, preparation of the EMR samples cannot be effectively made in standard 3D technology.

The comparison of experimental data with FEM simulations is presented. It proves the utility of the finite element method as a tool for the optimization of EMR sensor parameters.

\section{Acknowledgements}

This work was supported by a Poznan University of Technology grant No DS 62-176/1

\section{References}

[1] S.A. Solin, Scientific American 291, 44, (2004).

[2] M. Oszwaldowski, S. El-Ahmar, J. Jankowski, J. Phys. D, submitted to publication.

[3] M. Holz, O. Kronenwerth, D. Grundler, Phys. Rev. B 67, 195312, (2003).

[4] M. Hoener, O. Kronenwerth, Ch. Heyn, D. Grundler, M. Holz, J. Appl. Phys. 99, 036102, (2006).

[5] M. Oszwaldowski, T. Berus, A. Borowska, M. Nowicki, R. Czajka, M. Zimniak, Phys. Stat. Sol. (c) 1, 351, (2004).

[6] J. Moussa, L.R. Ram-Mohan, A.C.H Rowe, S.A. Solin, J. Appl. Phys. 94, 1110, (2003).

[7] M. Holz, O. Kronenwerth, D. Grundler, Appl. Phys. Lett. 83, 3344, (2003).

[8] M. Holz, O. Kronenwerth, D. Grundler, Appl. Phys. Lett. 86, 072513, (2005).

[9] M. Holz, O. Kronenwerth, D. Grundler, Appl. Phys. Lett. 87, 172501, (2005).

[10] J. Sun, J. Kosel, J. Supercond. Nov. Magn. in press DOI 10.1007/s10948-011-1256-8, (2011). 